\title{
Majoration du premier zéro de la fonction zêta de Dedekind
}

\author{
par \\ SAmi OMAR (Talence)
}

1. Introduction et notations. Soit $K$ un corps de nombres de degré $n$, de signature $\left(r_{1}, r_{2}\right)$ et de discriminant $d_{K}$. Dans [Od], A. M. Odlyzko évoque le problème de savoir l'ordre de grandeur du premier zéro de la fonction zêta de Dedekind. Dans cette direction, une conjecture a été énoncée dans [To] qui dit que la hauteur du premier zéro est majorée par $C / \ln \left(\left|d_{K}\right|\right)$ où $C$ est une constante positive qui ne dépend que de $n$. L'idée de cette dernière inégalité provient d'un théorème de densité (sous GRH) dû a S. Lang [La1]. Malgré les progrés numériques sur la question (voir [Om] et [To]), nous ne sommes toujours pas en mesure de confirmer expérimentalement cette conjecture. Cependant nous disposons d'un résultat théorique dû à A. Neugebauer [Ne1], [Ne2] qui montre que la hauteur du premier zéro est majorée par $C / \ln \ln \ln \left(\left|d_{K}\right|\right)$.

Dans ce qui suit nous donnerons une amélioration de cette inégalité qui sous $(\mathrm{GRH})$ aboutit à la majoration $C / \ln \ln \left(\left|d_{K}\right|\right)$. L'outil crucial de la preuve, comme nous le verrons, sont les formules explicites de Weil.

Dans la suite, la notation « réfère à une constante absolue alors que la notation $\ll_{n}$ réfère à une constante qui dépend uniquement de $n$.

2. Formules Explicites de Weil. Soit $F$ une fonction réelle d'une variable réelle qu'on peut supposer paire et qui vérifie les conditions (A) et (B) suivantes [La2] :

(A) $F$ est continue et continuement dérivable sur $\mathbb{R}$ sauf en un nombre fini de points $a_{i}$ où $F(x)$ et sa dérivée $F^{\prime}(x)$ n'ont que des discontinuités de première espèce pour lesquelles $F$ vérifie la condition de la moyenne (i.e. $\left.F\left(a_{i}\right)=\frac{1}{2}\left(F\left(a_{i}+0\right)+F\left(a_{i}-0\right)\right)\right)$.

(B) Il existe $b>0$ tel que $F(x)$ et $F^{\prime}(x)$ sont des $O\left(e^{-(1 / 2+b)|x|}\right)$ au voisinage de $\infty$.

2000 Mathematics Subject Classification: Primary 11R42. 
La transformée de Mellin de $F$ :

$$
\Phi(s)=\int_{-\infty}^{\infty} F(x) e^{(s-1 / 2) x} d x
$$

est alors holomorphe dans toute bande $-a \leq \sigma \leq 1+a$ où $0<a<b, a<1$ et on a le résultat dû à Weil $[\mathrm{Po}]$ :

ThÉORÈme 1 (Weil). Soit $F$ vérifiant les conditions $(\mathrm{A})$ et $(\mathrm{B})$ ci-dessus avec $F(0)=1$. Alors la somme $\sum \Phi(\varrho)$ étendue sur les zéros $\varrho=\beta+i \gamma$ non triviaux de $\zeta_{K}(s)$ avec $|\gamma|<T$ a une limite quand $T$ tend vers l'infini et sa somme est donnée par la formule:

$$
\begin{aligned}
\sum_{\varrho} \Phi(\varrho)= & \Phi(0)+\Phi(1)-2 \sum_{\mathfrak{p}, m} \frac{\ln (N(\mathfrak{p}))}{N(\mathfrak{p})^{m / 2}} F(m \ln (N(\mathfrak{p}))) \\
& +\ln \left(\left|d_{K}\right|\right)-n[\ln (2 \pi)+\gamma+2 \ln (2)]-r_{1} J(F)+n I(F)
\end{aligned}
$$

avec

$$
J(F)=\int_{0}^{\infty} \frac{F(x)}{2 \operatorname{ch}(x / 2)} d x, \quad I(F)=\int_{0}^{\infty} \frac{1-F(x)}{2 \operatorname{sh}(x / 2)} d x
$$

et $\gamma=0.57721566 \ldots$ désigne la constante d'Euler.

Remarque. On a

$$
\Phi(0)+\Phi(1)=4 \int_{0}^{\infty} F(x) \operatorname{ch}(x / 2) d x .
$$

Si $\widehat{F}$ désigne la transformée de Fourier de $F$ alors sous l'hypothèse de Riemann généralisée on a $\Phi(\varrho)=\widehat{F}(t)$ où $\varrho=1 / 2+i t$.

3. Estimation du premier zéro de la fonction zêta de Dedekind. Dans un premier temps on cherche à majorer la multiplicité d'un éventuel zéro de la fonction zêta de Dedekind au point 1/2 (cf. [Me] pour un résultat analogue sur les courbes elliptiques) :

Proposition 1. Soit r la multiplicité d'un éventuel zéro de la fonction $\zeta_{K}(s)$ au point $1 / 2$. Alors on a la majoration

$$
r \ll \frac{\ln \left(\left|d_{K}\right|\right)}{\ln \ln \left(\left|d_{K}\right|\right)} .
$$

Si on accepte la validité de la conjecture d'Artin sur la simplicité des zéros des séries $L$ d'Artin [Mu-Mu], on voit que $r$ est majoré par le nombre de caractères et ne dépend que de $n$.

Dans la preuve de la proposition 1, on suit une démarche similaire à celle de Ram Murty [Mu] qui donne une majoration de la multiplicité de $1 / 2$ comme zéro d'une série $L$ de Dirichlet. 
Preuve (de la proposition 1 ). Soit $F$ définie par

$$
F(x)= \begin{cases}1-|x| & \text { si }|x| \leq 1 \\ 0 & \text { sinon. }\end{cases}
$$

Alors $F$ vérifie les conditions du théorème 1 et on a

$$
\widehat{F}(u)=\left(\frac{2 \sin (u / 2)}{u}\right)^{2} .
$$

Si on pose $F_{T}(x)=F(x / T)$ alors $\widehat{F}_{T}(u)=T \widehat{F}(T u)$.

En appliquant maintenant les Formules Explicites à la fonction $F_{T}$, on obtient les inégalités

$$
\begin{aligned}
& r T \leq 4 \int_{0}^{T} \operatorname{ch}(x / 2) d x+\ln \left(\left|d_{K}\right|\right)+n \int_{0}^{T} \frac{x}{2 T \operatorname{sh}(x / 2)} d x, \\
& r T \leq e^{T / 2}+\ln \left(\left|d_{K}\right|\right)+n .
\end{aligned}
$$

Si on pose $T=2 \ln \ln \left(\left|d_{K}\right|\right)$, il s'ensuit que

$$
r \leq \frac{\ln \left(\left|d_{K}\right|\right)}{\ln \ln \left(\left|d_{K}\right|\right)}+\frac{n}{2 \ln \ln \left(\left|d_{K}\right|\right)} .
$$

Comme $n \ll \ln \left(\left|d_{K}\right|\right)$ (inégalité d'Odlyzko), on a $r \ll \ln \left(\left|d_{K}\right|\right) / \ln \ln \left(\left|d_{K}\right|\right)$.

ThÉORÈme 2. Soit h la hauteur du premier zéro différent de $1 / 2$ de la fonction zêta de Dedekind d'un corps de nombres $K$ de discriminant $d_{K}$. Alors on a l'estimation

$$
h \ll_{n} \frac{1}{\ln \ln \left(\left|d_{K}\right|\right)} .
$$

Corollaire 1. Si on prend une suite de corps de nombres pour lesquels $n$ est fixe et $d_{K}$ tend vers $\infty$ alors le premier zéro tend vers $1 / 2$.

Pour démontrer le théorème 2 , on a besoin de trois lemmes :

LEMme 1. Soit $F$ la fonction paire à support compact définie sur $[0, \infty]$ par

$$
F(x)= \begin{cases}(1-x) \cos (\pi x)+\frac{3}{\pi} \sin (\pi x) & \text { si } 0 \leq x \leq 1, \\ 0 & \text { sinon. }\end{cases}
$$

Alors $F$ vérifie les conditions du théorème 1 et

$$
\widehat{F}(u)=\left(2-\frac{u^{2}}{\pi^{2}}\right)\left[\frac{2 \pi}{\pi^{2}-u^{2}} \cos (u / 2)\right]^{2} .
$$

Preuve. On pourra vérifier que $F(x)$ s'écrit en termes de la fonction d'Odlyzko $G[\mathrm{Po}]$ et de sa dérivée seconde. En effet $F(x)=2 G(x)+\frac{1}{\pi^{2}} G^{\prime \prime}(x)$. 
Lemme 2. Si on pose $F_{T}(x)=F(x / T)$ avec $F$ comme au lemme 1 alors on a l'estimation suivante de la somme sur les idéaux premiers :

$$
\left|\sum_{\mathfrak{p}, m} \frac{\ln (N(\mathfrak{p}))}{N(\mathfrak{p})^{m / 2}} F_{T}(m \ln (N(\mathfrak{p})))\right| \ll n^{2} e^{T / 2} .
$$

Preuve. On a

$$
\left|\sum_{\mathfrak{p}, m} \frac{\ln (N(\mathfrak{p}))}{N(\mathfrak{p})^{m / 2}} F_{T}(m \ln (N(\mathfrak{p})))\right| \leq 2 \sum_{N(\mathfrak{p})^{m} \leq e^{T}} \frac{\ln (N(\mathfrak{p}))}{N(\mathfrak{p})^{m / 2}} \leq 2 n^{2} \sum_{p^{m} \leq e^{T}} \frac{\ln (p)}{p^{m / 2}} .
$$

On introduit d'une manière naturelle les fonctions arithmétiques $\Lambda(n)$ de Mangoldt et $\Psi(x)=\sum_{n \leq x} \Lambda(n)$ de Chebyshev. On réécrit alors la dernière somme :

$$
\sum_{p^{m} \leq e^{T}} \frac{\ln (p)}{p^{m / 2}}=\sum_{n \leq e^{T}} \frac{\Lambda(n)}{\sqrt{n}}=\int_{1}^{e^{T}} \frac{1}{\sqrt{x}} d \Psi(x) .
$$

Une intégration par parties de cette dernière intégrale donne

$$
\int_{1}^{e^{T}} \frac{1}{\sqrt{x}} d \Psi(x)=\frac{\Psi\left(e^{T}\right)}{e^{T / 2}}+\frac{1}{2} \int_{1}^{e^{T}} \frac{\Psi(x)}{x^{3 / 2}} d x .
$$

Or on sait que d'après le théorème de Shapiro [Ap] on a $\Psi(x) \ll x$, ce qui montre que

$$
\sum_{p^{m} \leq e^{T}} \frac{\ln (p)}{p^{m / 2}} \ll e^{T / 2} .
$$

Lemme 3. Soient $A, B, C$ trois constantes réelles positives vérifiant $C>$ 2B. Si $T>0$ vérifie $A T+B e^{T / 2} \geq C$, alors

Preuve. Par l'absurde.

$$
T \geq \varepsilon \ln (C), \quad \text { où } \varepsilon=\min \left(\frac{C}{2 A \ln (C)}, \frac{2 \ln (C /(2 B))}{\ln (C)}\right) .
$$

Preuve du théorème 2. On applique cette fois-ci les Formules Explicites à la fonction $F_{T}(x)=F(x / T)$ où $F$ est la fonction définie dans le lemme 1 ; et on pose $T=\sqrt{2} \pi / h$ où $h$ est la hauteur du premier zéro différent de $1 / 2$ de la fonction $\zeta_{K}(s)$. On obtient alors l'inégalité

$$
\begin{aligned}
\frac{8}{\pi^{2}} r T \geq & \Phi_{T}(0)+\Phi_{T}(1)-2 \sum_{\mathfrak{p}, m} \frac{\ln (N(\mathfrak{p}))}{N(\mathfrak{p})^{m / 2}} F_{T}(m \ln (N(\mathfrak{p}))) \\
& +\ln \left(\left|d_{K}\right|\right)-n[\ln (2 \pi)+\gamma+2 \ln (2)]-r_{1} J\left(F_{T}\right)+n I\left(F_{T}\right)
\end{aligned}
$$

il est facile de vérifier que

$$
\left|\Phi_{T}(0)+\Phi_{T}(1)\right| \ll e^{T / 2}
$$

et que les intégrales $J\left(F_{T}\right)$ et $I\left(F_{T}\right)$ sont bornées quand $T$ est grand. En 
se servant maintenant du lemme 2, l'inégalité (2) donne alors une inégalité comme celle du lemme 3 avec

$$
A=c_{1} \frac{\ln \left(\left|d_{K}\right|\right)}{\ln \ln \left(\left|d_{K}\right|\right)}, \quad C=\ln \left(\left|d_{K}\right|\right),
$$

où $c_{1}$ est tel que $r<c_{1} \frac{\pi^{2}}{8} \cdot \frac{\ln \left(\left|d_{K}\right|\right)}{\ln \ln \left(\left|d_{K}\right|\right)}$ (ceci est assuré grâce à la proposition 1$)$.

Finalement avec les constantes ci-dessus, on obtient

$$
\varepsilon=\min \left(\frac{1}{2 c_{1}}, 1-\frac{\ln (2 B)}{\ln \ln \left(\left|d_{K}\right|\right)}\right),
$$

et le résultat du théorème 2 découle facilement du lemme 3.

\section{Références}

[Ap] T. Apostol, Introduction to Analytic Number Theory, Springer, 1976.

[La1] S. Lang, On the zeta function of number fields, Invent. Math. 12 (1971), 337-345.

[La2] - Algebraic Number Theory, Addison-Wesley, 1968.

[Me] J.-F. Mestre, Courbes elliptiques et formules explicites, dans : Seminar on Number Theory, Paris, 1981-82, Progr. Math. 38, Birkhäuser, 1983, 179-187.

[Mu] M. R. Murty, Simple zeros of L-functions, dans : Number Theory, R. Mollin (ed.), de Gruyter, 1989, 427-439.

[Mu-Mu] M. R. Murty and V. K. Murty, Non-vanishing of L-Functions and Applications, Birkhäuser, 1997.

[Ne1] A. Neugebauer, On the zeros of the Dedekind zeta function near the real axis, Funct. Approx. Comment. Math. 16 (1988), 165-167.

[Ne2] - On zeros of zeta functions in low rectangles in the critical strip, Ph.D. thesis, A. Mickiewicz University, Poznań, Poland, 1985.

[Od] A. M. Odlyzko, Bounds for discriminants and related estimates for class numbers, regulators and zeros of zeta functions: A survey of recent results, Sém. Théor. Nombres Bordeaux 2 (1990), 119-141.

[Om] S. Omar, Localization of the first zero of the Dedekind zeta function, Math. Comp., à paraître.

[Po] G. Poitou, Sur les petits discriminants, Sém. Delange-Pisot-Poitou, 18e année, 1976/77, no. 6 .

[To] E. Tollis, Zeros of Dedekind zeta functions in the critical strip, Math. Comp. 66 (1997), 1295-1321.

Laboratoire d'Algorithmique Arithmétique

Université Bordeaux I

351 cours de la Libération

F-33405 Talence Cedex, France

E-mail: omar@math.u-bordeaux.fr 